\title{
Functional Significance of Sit-to-stand by Hip Abduction with External Rotation Angle
}

\author{
Takaki Kafuku ${ }^{1,3}$, Hiromi Fujii²,3, Yoko Kimura1,3, Miyuki Fujikura ${ }^{1,3}$, \\ Emiko Fukuda2,3 \\ ${ }^{1}$ Department of Occupational Therapy, Tohoku Medical College \\ 2 Department of Occupational Therapy, Yamagata Prefectural University of Health Sciences \\ ${ }^{3}$ Area of Occupational Therapy, Graduate School of Health Sciences, Yamagata Prefectural University of Health Sciences
}

\begin{abstract}
The principal aim of this study was to analyze the effects of differences in hip abduction and external rotation angles on sit-to-stand (STS) movements. Hip abduction and external rotation angles of 0 deg (closed legs parallel to each other) and $45 \mathrm{deg}$ (open legs) of 27 healthy participants were compared. Analysis parameters were the center of pressure, the ground reaction force, and the activities of fourteen muscles using surface electromyography. The center of pressure parameter revealed that standing with open legs requires less anterior-posterior displacement during STS movement. The open leg movement showed that ground reaction force differed slightly for right and left sides. Muscle activity of the erector spinae and tibialis anterior decreased with the STS movement with open legs. In contrast, muscle activity of the adductor longus and the biceps femoris increased.
\end{abstract}

Key words: sit-to-stand movement, center of pressure, the ground reaction forces, electromyogram, hip angle

(Asian J Occup Ther 10: 1-8, 2014)

\section{Introduction}

Japanese homes typically have a $20 \mathrm{~cm}$ step in the entrance hall (Research Institute of Human Engineering for Quality Life, 2002, Chapter 7), where people almost always put on and take off shoes. Most adults can put on and take off shoes in a standing position, but it is necessary for functionally limited elderly to sit down on the $20 \mathrm{~cm}$ step. Few elderly people can rise from a $20 \mathrm{~cm}$ high step without a footstool and handrails. Therefore, the daily life of such elderly is limited without access to a footstool and handrails. Clinically, the authors fixed the hips of the elderly at an angle of bilateral symmetry. Then the sit-to-stand (STS) movement was attempted from this position. Results show that, for hip abduction and external rotation angles of $0 \mathrm{deg}$ (closed legs parallel to each other; closed legs) the elderly were unable to stand up, but they were able to do so with hip abduction and external rota-

Received: 6 August 2012, Accepted: 26 February 2013

Corresponding to: Hiromi Fujii, Department of Occupational Therapy, Yamagata Prefectural University of Health Sciences, 260 Kamiyanagi,

Yamagata 990-2212, Japan

e-mail: hfujii@yachts. ac.jp

(C2014 Japanese Association of Occupational Therapists tion angles of $45 \mathrm{deg}$ (open legs). We concluded, therefore, that abduction and external rotation angles of the hip had positive effects on STS movement from a low step.

Factors affecting STS movement include chair seat height (Arborelius, Wretenberg \& Lindberg, 1992; Janssen, Bussmann \& Stam, 2002; Kuo, Tully \& Galea, 2010; Schenkman, Hughes, Samsa \& Studenski, 1996; Yamada \& Demura, 2004), use of armrests or arms (Alexander, Schultz \& Warwick, 1991; Arborelius et al., 1992; Eriksrud \& Bohannon, 2003; Etnyre \& Thomas, 2007; Janssen et al., 2002; Leung \& Chang, 2009; Schultz, Alexander \& Ashton-Miller, 1992), use of handrails (O'Meara \& Smith, 2005; O'Meara \& Smith, 2006), foot positioning (Janssen et al., 2002; Khemlani, Carr \& Crosbie, 1999; Lecours, Nadeau, Gravel \& Teixera-Salmela, 2008; Vander Linden, Brunt \& McCulloch, 1994), trunk positioning (Schenkman, Berger, Riley, Mann \& Hodge, 1990), STS movement speed (Bieryla, Anderson \& Madigan, 2009; Hanke, Pai \& Rogers, 1995; Pai \& Rogers, 1990; Vander Linden et al., 1994), age (Alexander et al., 1991; Lundin, Grabiner \& Jahnigen, 1995; Schultz et al., 1992; Zijlstra, Bisseling, Schlumbohm \& Baldus, 2010), and hip angles (Gotoh, Takada \& Suehiro, 2002). Nevertheless, no report describes kinematic differences in hip abduction and ex- 
ternal rotation angles on the STS movement. This study was intended define basic kinematic differences between open and closed legs in the STS movement.

\section{Methods}

\section{Participants}

Participants were 27 healthy volunteers (20 males and 7 females) ranging in age from 20-41 years (mean 22.3; standard deviations: SD 4.5), in weight from 39-100 $\mathrm{kg}$ (mean 62.6; SD 2.8), and in height from $152-183 \mathrm{~cm}$ (mean 167.3; SD 1.5). All participants indicated that the open legs position was easier for standing up than that of closed legs. No participants had diseases or injuries which could impair STS movement.

All participants gave their informed consent to the experimental procedure, which was approved by the Ethics Committee of Yamagata Prefectural University of Health Sciences, Yamagata, Japan.

\section{Instrumentation and data acquisition}

Fig. 1a (1), b (1) shows the start position of the experiment. The stool height was $50 \%$ of the length from the floor to the caput fibulae for closed legs $(17-25 \mathrm{~cm})$. The distance between the right and left heels was the same as the distance between the two acromia. Each participant comfortably positioned the neck and trunk. The STS movement speed was self-selected. Participants were instructed to fold their arms across their chest to remove any upper limb influence. The end point of the experiment was a standing position with complete extension of knee and hip joints.

The analysis parameters were the center of pressure (COP), the ground reaction forces (GRF), and electromyograms (EMG) of fourteen muscles.

COP and the magnitude of the right and left GRF were recorded using a sampling frequency of $50 \mathrm{~Hz}$ with two force plates (Kineto Gravicorder G-7100; Anima Corp, Tokyo). Low pass was filtered with a cutoff frequency of $50 \mathrm{~Hz}$. COP was calibrated after setting the two force plates. Participants were positioned with both legs and the buttocks on each force plate.

STS movement images were recorded from the front and lateral sides at 30 frames per second using two digital video cameras (NV-GS300; Panasonic Inc, Osaka).

EMGs of the erector spinae (ES), gluteus maximus (GMA), vastus lateralis (VL), adductor longus (AL), biceps femoris (BF), tibialis anterior (TA), and gastrocnemius (GC) were recorded with one pair of disposable surface electrodes (L-600C; Nihon Kohden Corp, Tokyo), attached longitudinally $2 \mathrm{~cm}$ apart on the central part of the contracted muscle belly. EMGs were amplified, band pass filtered $(10-500 \mathrm{~Hz})$, sampled at 1,000
$\mathrm{Hz}$, and fed into the computer (Teraview; Gigatex Ltd, Osaki). Then they were integrated (rectified and averaged) using a raw EMG (EMG integration program, Gigatex, Ltd., Osaki).

The movement pictures, the COP, GRF, and EMG data were recorded using Teraview.

\section{Procedure}

Participants performed sufficient practice trials in closed and open leg positions before the data collection. Data were collected from three trials under each condition. Participants were given adequate rest between trials to avoid fatigue.

Finally, according to methods of muscle testing (Hislop \& Montgomery, 2002, Chapter 3, 5), we recorded EMGs of maximum voluntary contraction of the measured muscles.

\section{Data analysis}

\section{Movement Phases}

Using video data STS movements were divided into three phases according to previous studies (Millington, Myklebust \& Shambes, 1992; Riley, Schenkman, Mann \& Hodge, 1991; Schenkman et al., 1990). During phase 1 (flexion-momentum phase), the buttocks left the chair seat (Fig. 1, (2)) as the head began to move horizontally. Phase 2 began after the buttocks separated from the chair seat and continued until ankle maximum dorsiflexion of the ankle. Phase 3 was from the ankle maximum dorsiflexion position to the complete standing position.

\section{Analysis of COP}

Analysis of COP consisted of two points. First, we studied COP at the initiation of movement in the anterior-posterior COP (COP-Y) and the medial-lateral COP (COP-X) direction. Second, we measured the maximum movement displacement of COP-Y and COP-X in each phase.

\section{Analysis of GRF}

The analysis of GRF examined the differences between the absolute values of the right and left load at identical points every $20 \mathrm{~ms}$. Furthermore, we calculated the mean difference in each phase.

\section{Analysis of EMG}

To facilitate intra-individual and inter-individual comparisons of EMG, a standardization procedure was performed. For standardization, the contraction level of the muscle was indicated by expressing the amplitude of the integrated EMG as a percentage of that produced by the maximum contraction (\%max). The \%max data were used as reference values in each phase. The analyzed data were the data in which EMG indicated no artifacts.

The analyzed COP, GRF, and EMG data compared the open legs with closed legs. Statistical processing used 
a

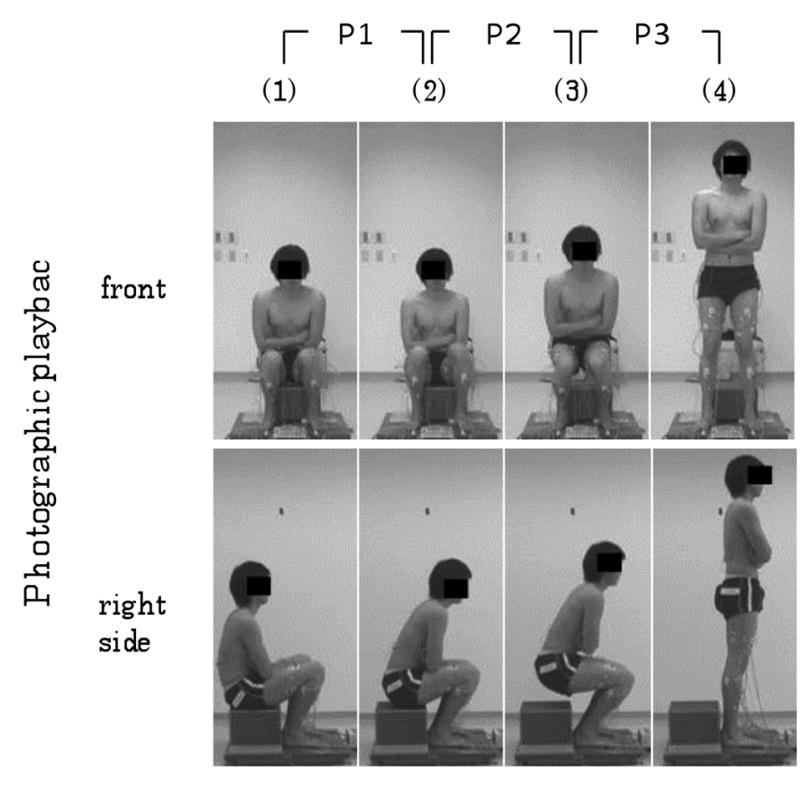

$\mathrm{b}$

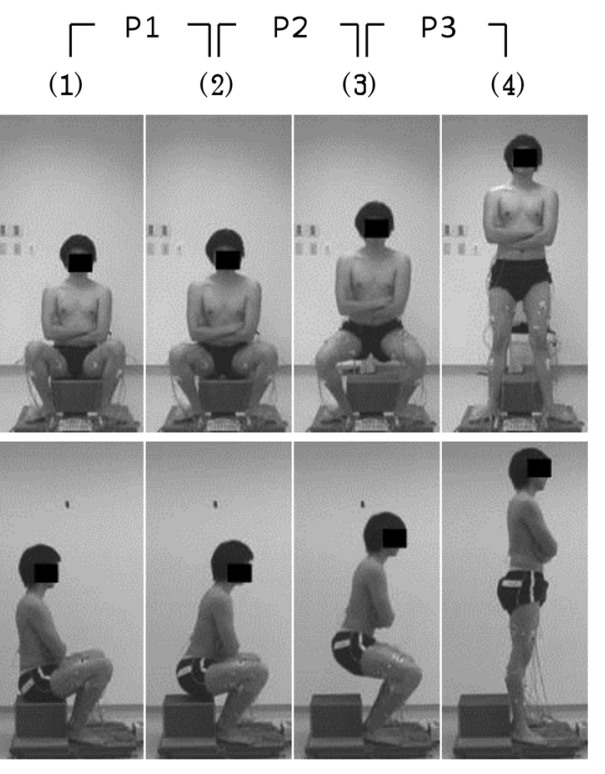

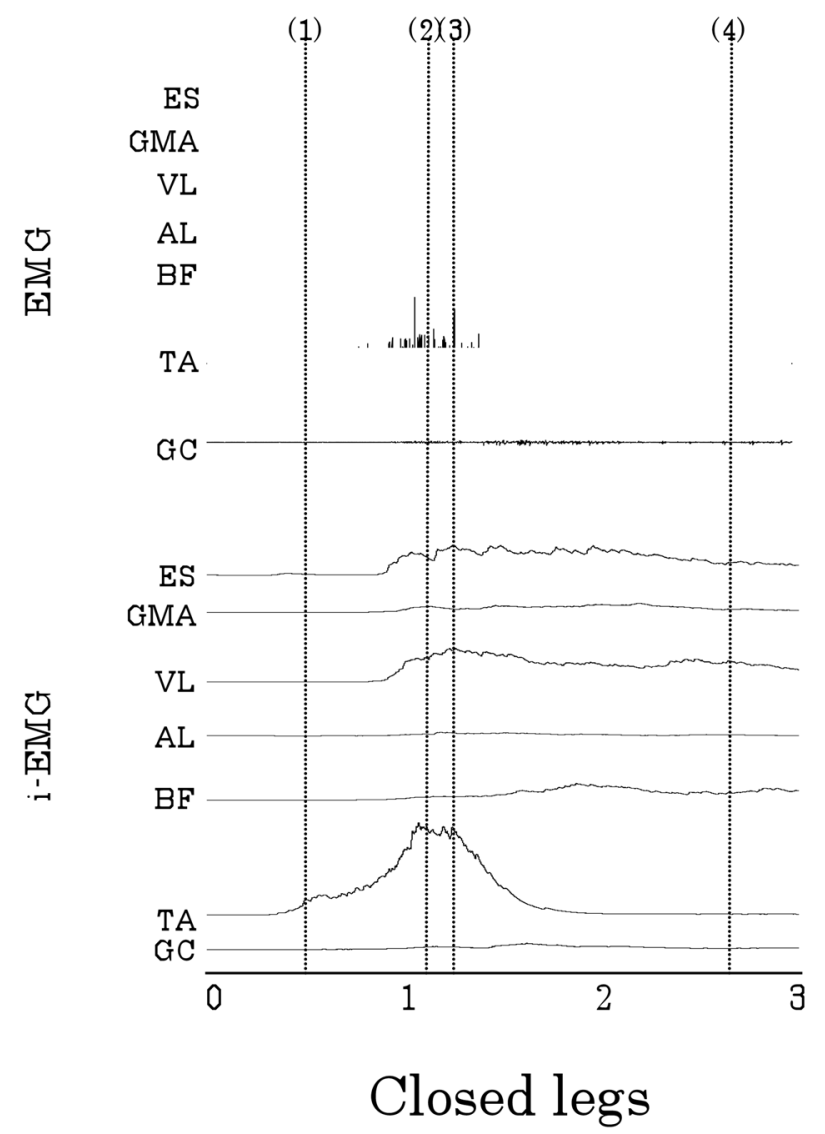

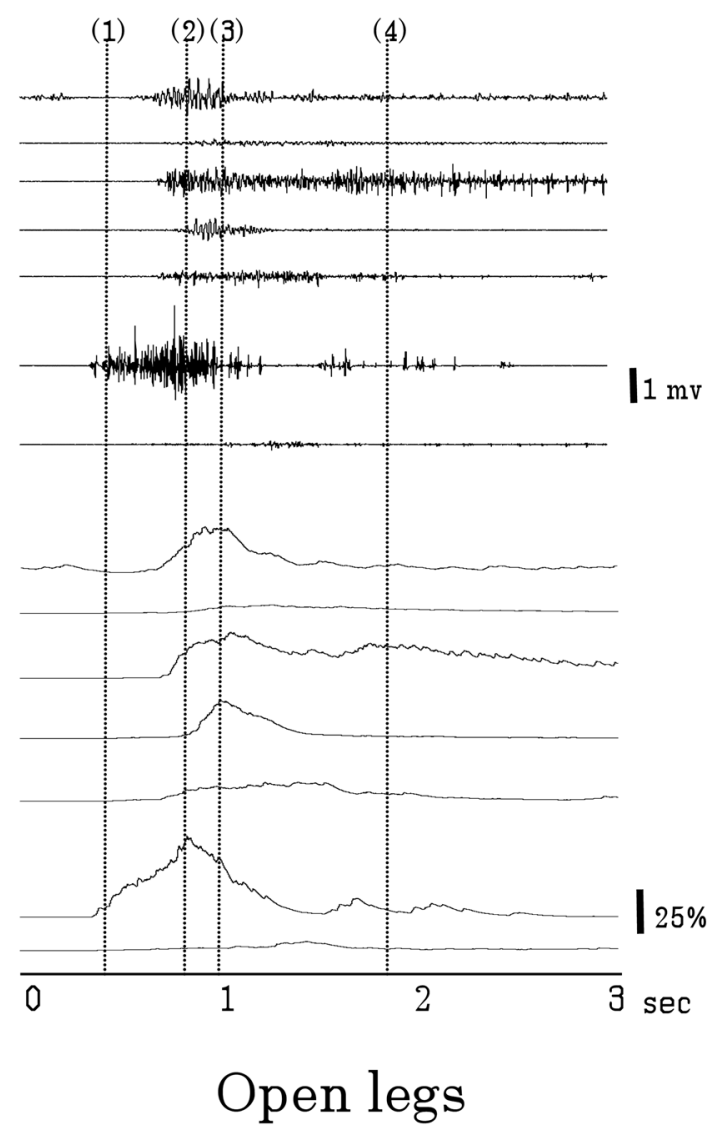

Fig. 1. Results of photographic playback, electromyogram (EMG), and integrated EMG (i-EMG). Vertical dotted lines indicate start (1), hip lift-off (2), maximal dosiflexion of the ankle joint (3), complete standing position (4). Calibration bar for EMGs and percentage of the amplitude produced by the maximum voluntary contraction (\%max). EMG activities of erector spinae (ES), gluteus maximus (GMA), vastus lateralis (VL), adductor longus (AL), biceps femoris (BF), tibialis anterior (TA), gastrocnemius (GC) during the STS movement with closed legs (a) and open legs (b). Phase 1 (P1), Phase 2 (P2), Phase 3 (P3). Abbreviations in this as well as Figs. 2-4, and Table 1. 


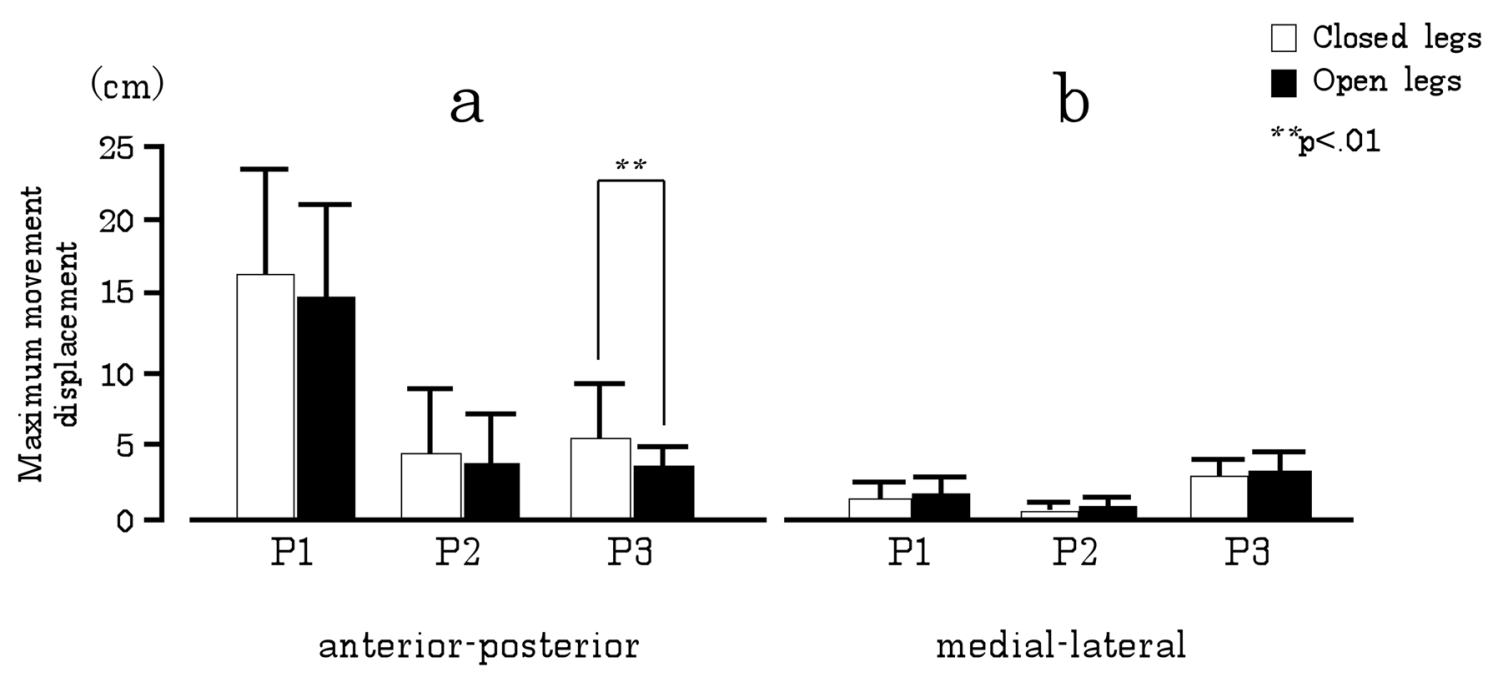

Fig. 2. The comparison of the maximum movement displacement during STS movement with anterior-posterior (a) and medial-lateral (b).

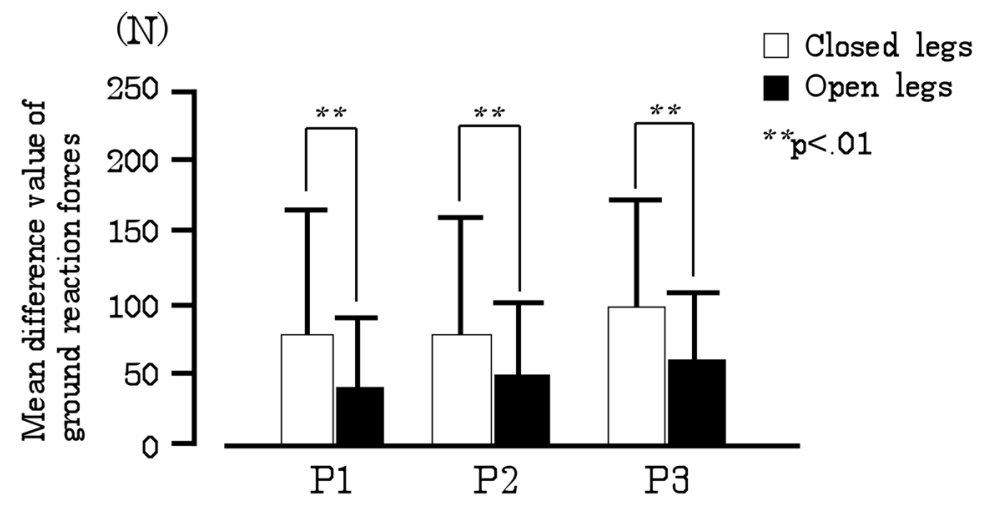

Fig. 3. The comparison of ground reaction forces.

student's $t$ tests, and a significance level of 0.05 was selected.

\section{Results}

\section{COP study}

The means and SD of COP-Y position at the start of movement with closed and open legs were $-14.48 \pm$ $5.80 \mathrm{~cm}$ and $-11.24 \pm 5.64 \mathrm{~cm}$, respectively. The COP-Y point at the start of movement with open legs was located significantly forward of the starting position $(\mathrm{p}<.01)$. COP-X position at the start of movement showed no statistically significant differences between closed and open legs.

The comparison of the maximum movement displacement of COP-Y in each phase is shown in Fig. 2a. In open legs, the maximum movement displacement of COP-Y was significantly less smaller than that of closed legs in phase $3(p<.01)$. For comparison, the maximum movement displacement of COP-X in each phase is shown in Fig. 2b. The maximum movement displacement of COP-X showed no statistically significant differences between closed and open legs in each phase.

GRF study

The comparison of GRF in each phase is shown in Fig. 3. In all phases, the average differences in right and left GRF were significantly smaller for open legs than closed legs $(\mathrm{p}<.01)$.

\section{EMG study}

Comparisons of the right and left \%max of each phase are shown in Table 1. Measurement of muscles found no significant difference between the right and left side. Comparison of right side \%max of the STS movement in closed and open legs is shown in Fig. 1 and Fig. 4. TA showed significantly decreased muscle activity in phase 2, although AL activity increased significantly in 
Table 1. The comparison of muscle activities in the left and right side limbs

\begin{tabular}{|c|c|c|c|c|c|c|c|c|c|c|c|c|c|c|c|c|c|c|}
\hline & \multicolumn{9}{|c|}{ Closed legs } & \multicolumn{9}{|c|}{ Open legs } \\
\hline & \multicolumn{3}{|c|}{ Phase 1} & \multicolumn{3}{|c|}{ Phase 2} & \multicolumn{3}{|c|}{ Phase 3} & \multicolumn{3}{|c|}{ Phase 1} & \multicolumn{3}{|c|}{ Phase 2} & \multicolumn{3}{|c|}{ Phase 3} \\
\hline & left & right & & left & right & & left & right & & left & right & & left & right & & left & right & \\
\hline ES & $19.0 \pm 9.8$ & $17.4 \pm 8.6$ & n.s & $29.3 \pm 13.0$ & $25.9 \pm 11.7$ & n.s & $15.4 \pm 7.7$ & $15.6 \pm 8.1$ & n.s & $17.6 \pm 8.4$ & $16.6 \pm 8.1$ & $\pi . s$ & $26.4 \pm 12.7$ & $26.4 \pm 13.0$ & $n . s$ & $13.1 \pm 8.4$ & $13.6 \pm 8.9$ & n.s \\
\hline GMA & $3.9 \pm 3.6$ & $5.2 \pm 7.1$ & n.s & $12.8 \pm 10.4$ & $14.9 \pm 17.1$ & n.s & $10.4 \pm 7.0$ & $11.6 \pm 7.1$ & n.s & $4.4 \pm 3.7$ & $5.0 \pm 4.9$ & n.s & $11.0 \pm 7.4$ & $11.8 \pm 10.5$ & n.s & $9.5 \pm 6.3$ & $10.8 \pm 7.1$ & n.s \\
\hline VL & $13.9 \pm 7.3$ & $13.2 \pm 5.6$ & ת.s & $51.9 \pm 22.9$ & $46.5_{ \pm} 24.9$ & n.s & $25.6 \pm 10.3$ & $25.7 \pm 10.5$ & $n .5$ & $15.1 \pm 8.7$ & $14.6 \pm 7.9$ & ת.s & $50.1 \pm 20.9$ & $46.7 \pm 22.9$ & ת.s & $26.6 \pm 11.2$ & $28.1 \pm 10.9$ & n.s \\
\hline $\mathrm{AL}$ & $9.8 \pm 8.2$ & $9.9 \pm 10.0$ & n.s & $16.1 \pm 13.2$ & $15.0 \pm 14.9$ & n.s & $6.9 \pm 4.2$ & $7.9 \pm 6.4$ & n.s & $10.1 \pm 11.3$ & $10.0 \pm 9.2$ & n.s & $21.3_{ \pm} 15.5$ & $22.6 \pm 21.2$ & n.s & $7.4 \pm 5.0$ & $8.6 \pm 7.6$ & n.s \\
\hline $\mathrm{BF}$ & $5.4 \pm 6.7$ & $6.0 \pm 8.7$ & n.s & $15.0 \pm 14.9$ & $13.8 \pm 16.0$ & n.s & $8.1 \pm 5.9$ & $9.8 \pm 9.8$ & n.s & $6.1 \pm 6.7$ & $6.8 \pm 8.0$ & n.s & $16.0 \pm 16.0$ & $15.9 \pm 15.7$ & n.s & $10.3 \pm 7.0$ & $12.1 \pm 9.8$ & n.s \\
\hline $\mathrm{TA}$ & $31.2 \pm 13.9$ & $31.6 \pm 15.6$ & n.s & $41.6 \pm 22.5$ & $40.2 \pm 21.8$ & n.s & $6.5_{ \pm} 4.7$ & $6.5 \pm 5.0$ & n.s & $30.7 \pm 16.4$ & $31.1 \pm 15.6$ & n.s & $32.9 \pm 23.1$ & $32.1 \pm 22.5$ & n.s & $6.4 \pm 5.3$ & $6.0 \pm 4.4$ & n.s \\
\hline $\mathrm{GC}$ & $4.2 \pm 3.4$ & $5.0 \pm 4.1$ & n.s & $6.1 \pm 4.5$ & $7.6 \pm 6.4$ & n.s & $7.0 \pm 5.3$ & $7.4 \pm 6.1$ & $n . s$ & $4.5_{ \pm} 4.1$ & $4.9 \pm 4.1$ & n.s & $6.9 \pm 5.5$ & $7.8 \pm 6.9$ & n.s & $7.5_{ \pm} 4.6$ & $8.3_{ \pm} 5.8$ & n.s \\
\hline
\end{tabular}

Percentage of the amplitude produced by the maximum contraction; \%max. ES; GMA; VL; AL; BF; TA; GC.
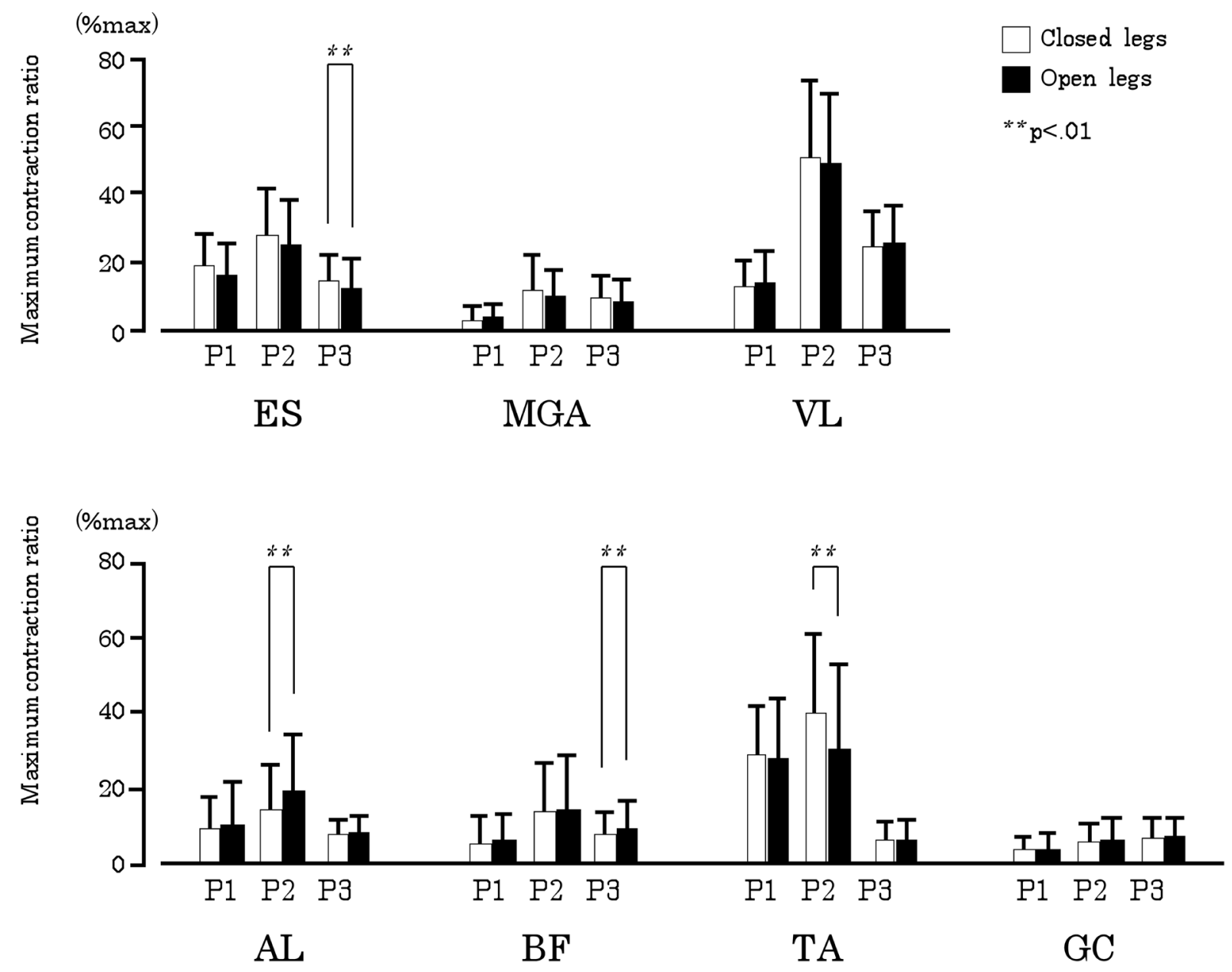

Fig. 4. The comparison of $\%$ max in each muscle.

open legs $(\mathrm{p}<.01)$. In phase 3 , ES showed significantly decreased muscle activity, and BF significantly increased for open legs $(\mathrm{p}<.01)$.

\section{Discussion}

Kinematic analysis of variable hip joint angles in healthy people has documented that the STS movements from hip external rotation positions increased to partial lumbar extension and pelvis anteversion (Gotoh et al., 
2002). In comparing sitting postures in Fig. 1 (right side, (1), a, b), open legs seemed to show greater increase of partial lumbar extension and pelvis anteversion than closed legs sitting in phase 1 . This suggests that the results of open legs resembled the above report. In this way, we speculate that COP-Y at the starting position with open legs was located significantly forward by pelvis anteversion. Moreover, another report stated that there was a center of force under the heels during the buttocks lift from the seat (Riley et al., 1991). In other words, COP of phase 1 is under the heels. This research shows that open legs with COP-Y at the time of initiation of STS movement were located in a forward position due to a short COP-Y displacement. It is speculated that this is one factor in making the STS movement easier.

Phase 2 was defined to be from after the buttocks lift off the chair seat to maximum dorsiflexion of the ankle (Schenkman et al., 1990). The center of mass is located in a maximum forward position at the time of ankle maximum dorsiflexion (Schenkman et al., 1990), and TA acted mainly in this ankle dorsiflexion (Gotoh et al., 2002). In addition, it was reported that STS movement from the hip external rotation angle decreased the anterior shift of the COP (Gotoh et al., 2002). Our results showed no significant differences in COP-Y between open and closed legs, but open legs decreased the anterior shift of the COP-Y in phase 2 (Fig. 2a, P2). Thus, our results indicate that there was slightly more ankle dorsiflexion with open legs than closed legs. Therefore, we suggest that TA showed significantly decreased muscle activity in open legs (Fig. 4). On the other hand, \%max of $\mathrm{AL}$ was significantly different between open legs and closed legs in this phase (Fig. 4). It isn't clear if activities of AL increased with open legs in phase 2. The analysis of video data did not show hip joint adduction from the start (Fig. 1b (2)) to the end (Fig. 1b (3)) in phase 2. It seems that an increase of AL activities does not affect hip joint adduction. AL arises mainly from the medial border of the pectineus muscle and from under the pubic tubercle, spreading outside from underneath, and partly attaches about halfway along the femur medial lip (Fagerson, 1998, p. 18; Jenkins, 2002, p. 288). Thus, AL influences adduction and flexion of the hip joint (Fagerson, 1998, p. 18; Jenkins, 2002, p. 288). Furthermore, AL acts as the hip external rotator (Moore, 1997, p. 103; Pansky, 1996, p. 255). In the present study, AL seems to maintain the hip joint external rotation position. It is suggested that $\mathrm{AL}$ acts as an external hip rotator at the hip joint abduction and external rotation position. Furthermore, AL under open legs position should be effective for approximately $20 \mathrm{~cm}$ heights in phase 2 of the STS movement.

In the present study, during phase 3 from the maximum ankle dorsiflexion position to the complete standing position, open legs had less displacement of COP-Y than closed legs, because open legs has less anterior-posterior displacement in phase 3. It has been reported that ES acts to suppress body bending in STS movement (Millington et al., 1992). In other words, muscle activity of ES decreases at the time of slight bending of the body. In comparing postures at the start of phase 3, open legs seemed to show less body bending than closed legs (see Fig.1, right side, (3), a, b). Therefore, in open legs with slight bending of the body, ES decreased muscle activities in comparison with phase 3 of closed legs. BF and VL showed increments of synchronous activities at phase 3 from the latter half of phase 1 (Fig. 1). In addition, BF of open legs increased \%max more than closed legs in phase 3. Anatomy textbooks describe $\mathrm{BF}$ as arising mainly from the ischial tuberosity of ischium (long head) and the lateral lip of femur (short head) and attaches to the head of the fibula. The long head of BF acts as an extensor, abductor and external rotator of hip joint, and a flexor of the knee joint. The short head of the BF acts as flexor of the knee joint. It was described that the knee joint is nearly fully extended, the long head of BF acts as an extensor of the hip joint (Kapandji, 2011, p. 44). In this study, the knee joint has to extend so that the BF acts as a hip extensor. The open legs knee extension angle was larger than closed legs at the end of phase 2 (see Fig. 1a(3), b(3)). It is suggested that activities of BF promote hip extension, and help antigravity movement of the body.

In kinematic studies, it has been reported that GRF of STS movement was asymmetric for both right and left limbs, and STS movements of healthy subjects could be completed with different distributions of the body weight on both feet, because healthy people have strong muscle power (Lundin et al., 1995). Our closed legs results were similar to the above report. However, GRF of open legs had right and left symmetry in all phases. It is speculated that the STS movement of open legs is effective for the standing up of the elderly who have decreased muscle power.

As mentioned above, the STS movement from the open legs position has anterior position of COP, short displacement distance of COP, and symmetrical GRF. Therefore there is less muscle activity of ES and TA with open legs in comparison with closed legs. Furthermore, AL must be active during phase 2, which must be very beneficial to fix the hip joint during the STS movement from open legs. The functional significance of the STS movement with open legs promotes hip and knee extension, and helps antigravity movement of the body.

\section{Study limitations}

This study analyzed the effects of differences in hip abduction and external rotation angles on the STS movements of young people. However, the relation of STS 
movements between young people and the functionally limited elderly is unclear. In the future, we would like to investigate the STS movement by hip abduction and external rotation angles in the elderly.

\section{Conclusion}

This study analyzed the influence of differences in hip abduction and external rotation angles on STS movement. The STS movement from open legs showed less trunk flexion, and it indicated that GRF differed slightly for right and left. In addition, muscle activity of ES and TA decreased with open legs. In contrast, muscle activity of $\mathrm{AL}$ and $\mathrm{BF}$ increased.

\section{References}

Alexander, NB., Schultz, AB., \& Warwick, DN. (1991). Rising from a chair: effects of age and functional ability on performance biomechanics. J Gerontol. 46, M91-M98. [Medline] [CrossRef]

Arborelius, UP., Wretenberg, P., \& Lindberg, F. (1992). The effects of armrests and high seat heights on lower-limb joint load and muscular activity during sitting and rising. Ergonomics. 35, 1377-1391. [Medline] [CrossRef]

Bieryla, KA., Anderson, DE., \& Madigan, ML. (2009). Estimations of relative effort during sit-to-stand increase when accounting for variations in maximum voluntary torque with joint angle and angular velocity. $J$ Electromyogr $\mathrm{Ki}$ nesiol. 19, 139-144. [Medline] [CrossRef]

Eriksrud, O., \& Bohannon, RW. (2003). Relationship of knee extension force to independence in sit-to-stand performance in patients receiving acute rehabilitation. Phys Ther. 83, 544-551. [Medline]

Etnyre, B., \& Thomas, DQ. (2007). Event standardization of sit-to-stand movements. Phys Ther. 87, 1651-1666. [Medline] [CrossRef]

Fagerson, TL. (1998). Anatomy and biomechanics. Fagerson T.L, The hip handbook (p. 16). Boston: Butterworth Heinemann.

Gotoh, A., Takada, T., \& Suehiro, K. (2002). Standing up. J Kansai Phys Ther. 2, 25-40. (in Japanese).

Hanke, TA., Pai, YC., \& Rogers, MW. (1995). Reliability of measurements of body center-of-mass momentum during sit-to-stand in healthy adults. Phys Ther. 75, 105-113, discussion 113-118. [Medline]

Hislop, HJ., \& Montgomery, J. (2002). Chapter 3, 5. Daniels and Worthingham's muscle testing, techniques of manual examination. 7th ed (pp. 33-40, pp. 181-241). Philadelphia: W.B. Saunders Company.

Janssen, WG., Bussmann, HB., \& Stam, HJ. (2002). Determinants of the sit-to-stand movement: a review. Phys Ther. 82, 866-879. [Medline]

Jenkins, DB. (2002). Chapter 16. Waltner, P, Hollinshead's functional anatomy of the limbs and back. 8th ed (p. 288).
Philadelphia: W.B. Saunders Company.

Kapandji, AI. (2011). Chapter 1. Lally, N, The Physiology of the Joints, Volume two: Lower Limb, 6nd ed (p. 44). London: Churchill Livingstone.

Khemlani, MM., Carr, JH., \& Crosbie, WJ. (1999). Muscle synergies and joint linkages in sit-to-stand under two initial foot positions. Clin Biomech (Bristol, Avon). 14, 236246. [Medline] [CrossRef]

Kuo, YL., Tully, EA., \& Galea, MP. (2010). Kinematics of sagittal spine and lower limb movement in healthy older adults during sit-to-stand from two seat heights. Spine. 35, E1-E7. [Medline] [CrossRef]

Lecours, J., Nadeau, S., Gravel, D., \& Teixera-Salmela, L. (2008). Interactions between foot placement, trunk frontal position, weight-bearing and knee moment asymmetry at seat-off during rising from a chair in healthy controls and persons with hemiparesis. J Rehabil Med. 40, 200-207. [Medline] [CrossRef]

Leung, CY., \& Chang, CS. (2009). Strategies for posture transfer adopted by elders during sit-to-stand and stand-to-sit. Percept Mot Skills. 109, 695-706. [Medline] [CrossRef]

Lundin, TM., Grabiner, MD., \& Jahnigen, DW. (1995). On the assumption of bilateral lower extremity joint moment symmetry during the sit-to-stand task. J Biomech. 28, 109 112. [Medline] [CrossRef]

Millington, PJ., Myklebust, BM., \& Shambes, GM. (1992). Biomechanical analysis of the sit-to-stand motion in elderly persons. Arch Phys Med Rehabil. 73, 609-617. [Medline]

Moore, NA. (1997). Chapter 3. Underdown, E.D, Mosby's USMLE step 1 reviews - anatomy (p. 103). St. Louis: Mosby.

O'Meara, DM., \& Smith, RM. (2005). Differences between grab rail position and orientation during the assisted sitto-stand for able-bodied older adults. J Appl Biomech. 21, 57-71. [Medline]

O'Meara, DM., \& Smith, RM. (2006). The effects of unilateral grab rail assistance on the sit-to-stand performance of older aged adults. Hum Mov Sci. 25, 257-274. [Medline] [CrossRef]

Pai, YC., \& Rogers, MW. (1990). Control of body mass transfer as a function of speed of ascent in sit-to-stand. Med Sci Sports Exerc. 22, 378-384. [Medline] [CrossRef]

Pansky, B. (1996). Unit Six. Review of gross anatomy. 6th ed (p. 225). Gavert, G, New York: McGraw Hill.

Research Institute of Human Engineering for Quality Life. (2002). Guidelines on daily living related apparatus and equipment design for elderly people. Retrieved from http://www.hql.jp/project/funcdb2000/guideline.ver.2/ nitijo-tekiyourei27.pdf (in Japanese).

Riley, PO., Schenkman, ML., Mann, RW., \& Hodge, WA. (1991). Mechanics of a constrained chair-rise. J Biomech. 24, 77-85. [Medline] [CrossRef]

Schenkman, M., Berger, RA., Riley, PO., Mann, RW., \& Hodge, WA. (1990). Whole-body movements during rising to standing from sitting. Phys Ther. 70, 638-648, discussion 648-651. [Medline]

Schenkman, M., Hughes, MA., Samsa, G., \& Studenski, S. 
(1996). The relative importance of strength and balance in chair rise by functionally impaired older individuals. $\mathrm{J} \mathrm{Am}$ Geriatr Soc. 44, 1441-1446. [Medline]

Schultz, AB., Alexander, NB., \& Ashton-Miller, JA. (1992). Biomechanical analyses of rising from a chair. J Biomech. 25, 1383-1391. [Medline] [CrossRef]

Vander Linden, DW., Brunt, D., \& McCulloch, MU. (1994). Variant and invariant characteristics of the sit-to-stand task in healthy elderly adults. Arch Phys Med Rehabil. 75, 653-660. [Medline] [CrossRef]
Yamada, T., \& Demura, S. (2004). Influence of the relative difference in chair seat height according to different lower thigh length on floor reaction force and lower-limb strength during sit-to-stand movement. J Physiol Anthropol Appl Human Sci. 23, 197-203. [Medline] [CrossRef]

Zijlstra, W., Bisseling, RW., Schlumbohm, S., \& Baldus, H. (2010). A body-fixed-sensor-based analysis of power during sit-to-stand movements. Gait Posture. 31, 272-278. [Medline] [CrossRef] 